\title{
Overlapping XAFS L Spectra of 3d Metals: A New Application of the Regularization Method
} \author{
Yu. Dedkov ${ }^{6}$, M. Fonine ${ }^{6}$, K. Baberschke ${ }^{4}$, H. Wende ${ }^{4}$ and Y. U. Idzerda ${ }^{7}$ \\ ${ }^{1}$ Institute of Metal Physics, RAS, GSP-170, 620219, Ekaterinburg, Russia \\ ${ }^{2}$ Udmurt State University, Izhevsk, Russia \\ ${ }^{3}$ Russian-German Laboratory at BESSY II, 12489 Berlin, Germany \\ ${ }^{4}$ Freie Universität Berlin, 14195 Berlin, Germany \\ ${ }^{5}$ Technische Universität Dresden, 01062 Dresden, Germany \\ ${ }^{6}$ Physikalisches Institut RWTH, Aachen D-52056, Germany \\ ${ }^{7}$ Montana State University, Bozeman, MT 59717-3840, USA
}

Yu. Babanov ${ }^{1,2}$, S. Kiryanov ${ }^{1}$, A. Sidorenko ${ }^{1}$, L. Romashev ${ }^{1}$, D. Vyalikh ${ }^{3,4}$, S. Molodtsov ${ }^{3,5}$, G. Guentherodt ${ }^{6}$, U. Ruediger ${ }^{6}$,

\begin{abstract}
$L$ XAFS spectra of polycrystalline Fe and Cr films are investigated by EXAFS and TEY (Total Electron Yield) techniques. A new method of obtaining local structure information from overlapping $L$ XAFS spectra for $3 \mathrm{~d}$ metals is proposed. Tikhonov regularization method of solving ill-posed problem is used. In contrast to the conventional methods (Fourier transformation and fitting procedures) this method does not demand any model assumption and any special procedure of deconvolution of $L_{1}-, L_{2}-, L_{3}$ - contributions. The parameters obtained from $L$ XAFS spectra of polycrystalline $\mathrm{Fe}$ and $\mathrm{Cr}$ films (interatomic distance and coordination number for the first and the second coordination shells) agree with the expected crystallographic values. Up to now the regularization method was applied only to nonoverlapping XAFS spectra.
\end{abstract}

\section{Introduction}

If the photon energy is high enough to excite an electron from an atomic core to the continuum, an edge-jump is found in the $\mathrm{X}$ ray absorption coefficient. The excitation thresholds $K, L_{1}, L_{2}, L_{3}$ correspond to the transition of $1 s_{1 / 2} \rightarrow \varepsilon p, 2 s_{1 / 2} \rightarrow \varepsilon p, 2 p_{1 / 2} \rightarrow$ $\varepsilon s, \varepsilon d, 2 p_{3 / 2} \rightarrow \varepsilon s, \varepsilon d$. The special feature of $L$ spectra of $3 \mathrm{~d}$ metals is overlapping. Fig. 1 shows model $L$ spectra (total one and separate contributions) for pure Fe calculated by FEFF8 [1].

We propose a new method of obtaining local structure information from the overlapping $L$ XAFS spectra for $3 \mathrm{~d}$ metals using Tikhonov's method of solving ill-posed problem [2]. Up to now this method was applied to nonoverlapping EXAFS spectra

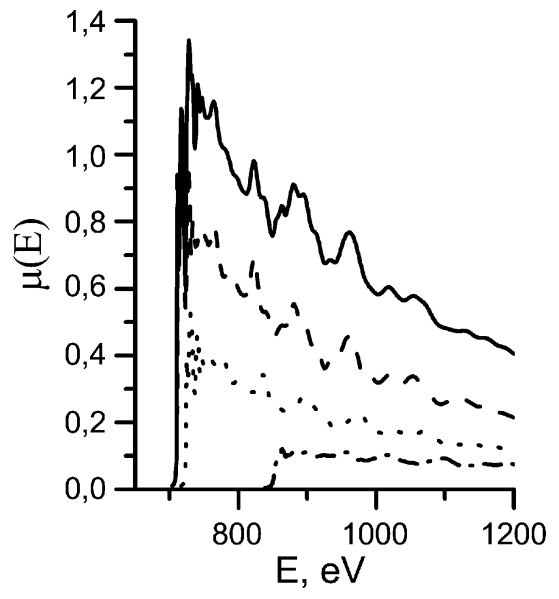

Fig. 1. FEFF8 simulation of $L$ spectra (the total one (solid line) and the separate contributions: $L_{3}$ (dash line), $L_{2}$ (dot line) and $L_{1}$ (dash dot line)) for pure Fe.
$[3,4]$. Recently a special procedure of deconvolution $L_{2}-$, $L_{3}$ - contributions [5] was used for obtaining local structure information.

\section{Mathematical algorithm}

For one component systems the atomic distribution is described by the pair correlation function (PCF) $g(r)$ which may be written as

$g(r)=\frac{1}{4 \pi \rho_{0}} \sum_{l} \frac{N_{l}}{r_{l}^{2} \sigma_{l} \sqrt{2 \pi}} \exp \left(-\frac{\left(r-r_{l}\right)^{2}}{2 \sigma_{l}^{2}}\right)$.

Here $\rho_{0}$ is atomic density, $N_{l}$ is coordination number for the $l$ shell, $\sigma_{l}$ is Debye-Waller factor for the $l$ shell, $r_{l}$ is position of the $l$ shell. The normalized oscillating part of the EXAFS signal $\chi(k)$ for the $K-$ spectrum is described as

$$
\begin{aligned}
\chi(k)= & 4 \pi \rho_{0} \int_{0}^{\infty} R(k) \frac{f(k, r)}{k} \exp \left(\frac{-2 r}{\lambda(k)}\right) \\
& \times \sin (2 k r+2 \delta(k)+\varphi(k, r)) g(r) \mathrm{d} r .
\end{aligned}
$$

Here $R(k)$ is reduction factor, $f(k, r)$ is a backscattering amplitude module, $\lambda(k)$ is the mean free path of a photoelectron, $\delta(k)$ is central atom phase shift and $\varphi(k, r)$ is a backscattering phase. All values are calculated by FEFF8 [1].

Using the dispersion laws for $L_{3}, L_{2}, L_{1}$ spectra one may conclude that $L$ spectra have the same $E$ space but different $k$ spaces (namely, $k_{1}, k_{2}, k_{3}$ ). The laws for connection of $k_{1}, k_{2}, k_{3}$ spaces is found as:

$$
\begin{aligned}
& k_{2}=f\left(k_{3}\right)=\sqrt{k_{3}^{2}+\left(E_{3}-E_{2}\right) / a}, \\
& k_{1}=h\left(k_{3}\right)=\sqrt{k_{3}^{2}+\left(E_{3}-E_{1}\right) / a},
\end{aligned}
$$

where $E_{3}, E_{2}, E_{1}$ are energies of $L_{3}, L_{2}, L_{1}$ edges, $a=$ $\hbar^{2} k^{2} /(2 m)=3.81$. Now the equation for the sum of $L_{1}, L_{2}, L_{3}$ EXAFS spectra in unified $k_{3}$ space may be written as [6]:

$$
\begin{aligned}
\chi_{\text {sum }}\left(k_{3}\right)= & W_{\mathrm{L} 3} \chi_{\mathrm{L} 3}\left(k_{3}\right)+W_{\mathrm{L} 2} \chi_{\mathrm{L} 2}\left(k_{2}=f\left(k_{3}\right)\right) \\
& +W_{\mathrm{L} 1} \chi_{\mathrm{L} 1}\left(k_{1}=h\left(k_{3}\right)\right),
\end{aligned}
$$

where $W_{\mathrm{L} 3}, W_{\mathrm{L} 2}, W_{\mathrm{L} 1}$ are weight coefficients used to account the contributions of each edge and calculated by FEFF8 [1].

For $L$ spectra the radial matrix elements are given by $M_{01}$ for $l=1 \rightarrow l=0$ transitions and $M_{21}$ for $l=1 \rightarrow l=2$ transitions. As shown in [7] for elements with $Z \geq 20$ the ratio $M_{01} / M_{21}$ 
is nearly independent of $k$, and the $p \rightarrow s$ contributions are practically unobservable, as $p \rightarrow d$ transitions are favored by a factor of 50. This means that all $M_{01}$ contributions in the integral equation can be neglected and hence the $L$ spectra can be analyzed in the same way as is done for the $K$ spectra.

The integral equation for overlapping $L$ spectra has the form:

$$
\begin{aligned}
& \chi\left(k_{3}\right)=4 \pi \rho_{0} \int_{0}^{\infty} \mathrm{d} r g(r) \\
& \quad \times\left\{W_{l 1} \frac{f_{1}\left(k_{1}, r\right)}{k_{1}} \exp \left(\frac{-2 r}{\lambda_{1}\left(k_{1}\right)}\right) \sin \left(2 k_{1} r+2 \delta_{1}\left(k_{1}\right)+\varphi_{1}\left(k_{1}, r\right)\right)\right. \\
& +W_{l 2} \frac{f_{2}\left(k_{2}, r\right)}{k_{2}} \exp \left(\frac{-2 r}{\lambda_{2}\left(k_{2}\right)}\right) \sin \left(2 k_{2} r+2 \delta_{2}\left(k_{2}\right)+\varphi_{2}\left(k_{2}, r\right)\right) \\
& \left.+W_{l 3} \frac{f_{3}\left(k_{3}, r\right)}{k_{3}} \exp \left(\frac{-2 r}{\lambda_{3}\left(k_{3}\right)}\right) \sin \left(2 k_{3} r+2 \delta_{3}\left(k_{3}\right)+\varphi_{3}\left(k_{3}, r\right)\right)\right\} .
\end{aligned}
$$

If we compare Eq. (2) for $K$ spectra and Eq. (5) for $L$ spectra we can see that in the last case we have the integral Fredholm equation with more complex kernel than in the first one. For $L$ spectra it is possible to use the regularization method without any modification. Details of solving the EXAFS ill-posed problem may be found in [2-4]. For multicomponent systems the theory is easy to generalize. It is possible to apply this approach not only for overlapping $L$ spectra but also for overlapping $K$ - $L$ spectra (for example, in the oxides $\mathrm{Fe}_{3} \mathrm{O}_{4}, \mathrm{Cr}_{2} \mathrm{O}_{3}$ ).

\section{Experimental details}

Two different techniques of experiment were used in this article - EXAFS in transmission mode and Total Electron Yield (TEY) technique.

TEY experiments were performed at room temperature using the facility of the RGBL beamline of BESSY II. The samples were polycrystalline $\mathrm{Fe}$ and $\mathrm{Cr}$ films. Fe film of thickness $\sim 300 \AA$ was evaporated under UHV conditions onto $\mathrm{W}(110)$ substrate and the measurements were performed immediately after deposition. Cr film of thickness $\sim 300-400 \AA$ was evaporated under UHV conditions onto $\mathrm{MgO}(100)$ substrate at the Institute of Metal Physics, RAS.

The low temperature (70K) EXAFS measurements were carried out in transmission mode at the U4B beamline of the National Synchrotron Light Source (NSLS) located at Brookhaven National Laboratory (BNL, USA). The sample was polycrystalline Fe film. This film with thickness of $500 \AA$ was evaporated under UHV conditions onto $\sim 1 \mu \mathrm{m}$ thick semitransparent parylene $\left(\mathrm{C}_{8} \mathrm{H}_{8}\right)_{n}$ substrate (100). To prevent the film from oxidising an additional $\mathrm{Al}$ cap layer was deposited on the film.

For obtaining $\chi(k)$ functions for $\mathrm{Fe}$ and $\mathrm{Cr}$ films from raw experimental data we use the conventional procedure [8]. We normalise the experimental spectrum on sum of $L_{3}, L_{2}, L_{1}$ edge jumps, but we do not remove $L_{1}$ edge jump from the experimental $\chi(k)$ because the value of this jump in comparing with the common jump is approximately 0.1 (see Fig. 1). It validates by the fact that there is no jump at $k \sim 6 \AA^{-1}$ in the experimental spectra in contrast with the model simulation at the temperature $70 \mathrm{~K}$ (Fig. 2).

\section{Discussion}

Fig. 2 shows experimental $\chi(k)$ functions for $\mathrm{Fe}$ and $\mathrm{Cr}$ films obtained from TEY measurements and for Fe film obtained from

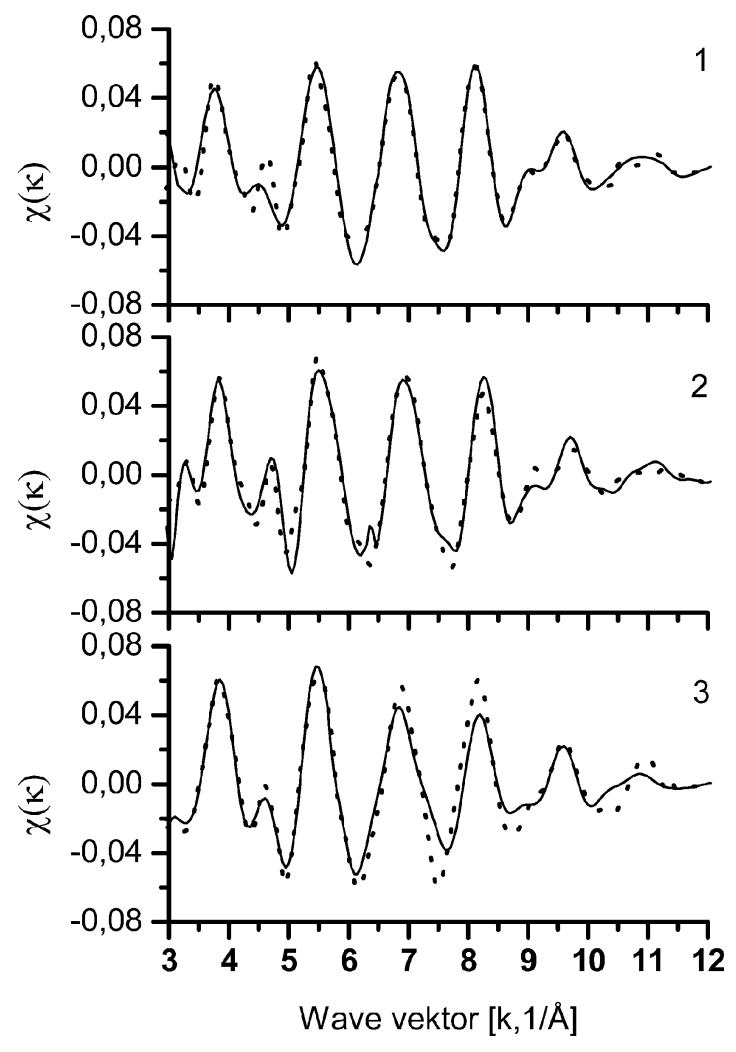

Fig. 2. Experimental EXAFS and TEY $\chi(k)$ spectra (dot line) in comparison with simulated functions by FEFF8 (solid lines) (1 - TEY spectra of Fe at $300 \mathrm{~K}$, 2 -EXAFS spectra of $\mathrm{Fe}$ at $70 \mathrm{~K}, 3$ - TEY spectra of $\mathrm{Cr}$ at $300 \mathrm{~K}$ ).

EXAFS measurements in transmission mode. The extraction of the $\chi(k)$ functions from raw data was used [3, 4]. TEY $\chi(k)$ functions were rather noisy due to low statistics so these curves were smoothed. All curves are compared with the model functions calculated by FEFF8 [1].

Applying the regularization method described [2-4], one can find the solutions which are shown on Fig. 3. The obtained results

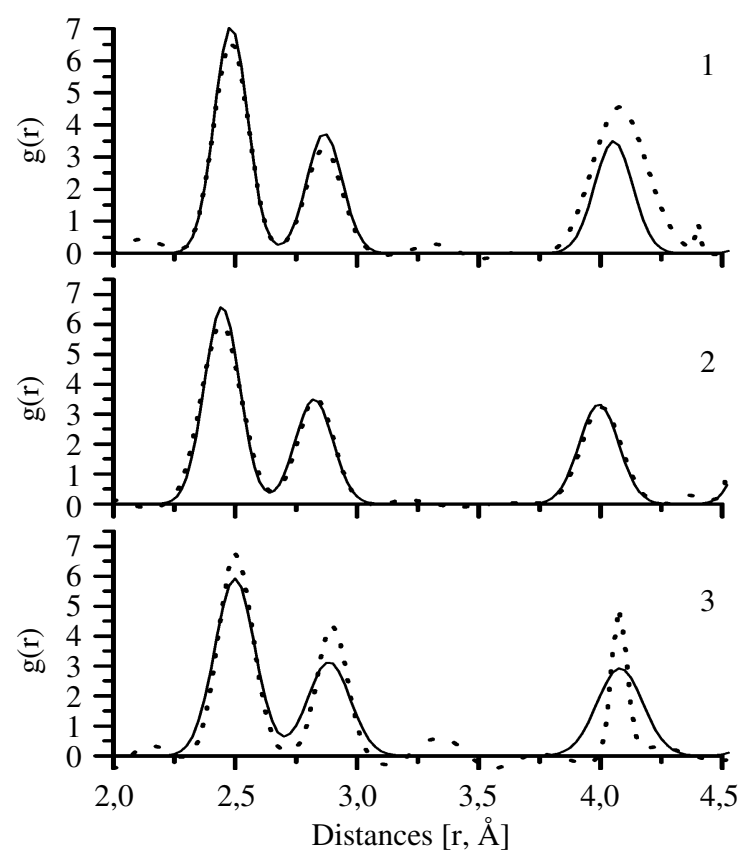

Fig. 3. Model PCF (solid line) and obtained (dot line) solution (1 - Fe at $300 \mathrm{~K}$, $2-\mathrm{Fe}$ at $70 \mathrm{~K}, 3-\mathrm{Cr}$ at $300 \mathrm{~K})$. 
Table I. Comparison of the model and experimental values obtained for the first and second shells of crystalline $\mathrm{Fe}$ and $\mathrm{Cr}$ (interatomic distance $r$ and nearest neighbors coordination number $N)$.

\begin{tabular}{|c|c|c|c|c|c|}
\hline \multirow[b]{2}{*}{ Edge } & \multirow[b]{2}{*}{ Type of experiment } & \multicolumn{2}{|c|}{$\begin{array}{c}1^{\text {st }} \text { coordination } \\
\text { shell }\end{array}$} & \multicolumn{2}{|c|}{$\begin{array}{c}2^{\text {nd }} \text { coordination } \\
\text { shell }\end{array}$} \\
\hline & & $r, \AA$ & $N$, at. & $r, \AA$ & $N$, at. \\
\hline \multirow[t]{4}{*}{$\mathrm{Fe}$} & Model $300 \mathrm{~K}$ & 2.483 & 8.00 & 2.867 & 6.00 \\
\hline & TEY & 2.482 & 7.87 & 2.865 & 5.38 \\
\hline & Model $70 \mathrm{~K}$ & 2.446 & 8.00 & 2.824 & 6.00 \\
\hline & EXAFS & 2.443 & 8.12 & 2.832 & 5.84 \\
\hline \multirow[t]{2}{*}{$\mathrm{Cr}$} & Model $300 \mathrm{~K}$ & 2.499 & 8.00 & 2.885 & 6.00 \\
\hline & TEY & 2.501 & 7.85 & 2.899 & 5.90 \\
\hline
\end{tabular}

are compared with model PCF functions calculated on the basis of the well known crystallographic data.

The following main characteristics of the peaks for the function $g(r)$ have been selected: interatomic distance $r$ and the nearest neighbors' coordination number $N$. These values were estimated according to the Gauss functions approximating the peaks of the solution and presented in Table I.

As it can be seen the main characteristics of the first and the second shells for crystalline $\mathrm{Fe}$ and $\mathrm{Cr}$ correspond to the crystallographic table values. There is a very high resolution in real space $r$ with comparing the Fourier transformation. On the curve of Fourier transformation it is impossible to distinguish the first and the second shells of BCC lattice.

It is necessary to note that during the solution of the inverse problem (2) the contribution of photoelectron multiple scattering on the nearest neighbours were not accounted. It seems than simultaneous solving of three $L$ spectra made the problem more stable.

\section{Conclusion}

The proposed new method of obtaining local structure information from overlapping XAFS spectra for $3 \mathrm{~d}$ metals shows its efficiency. In contrast to the conventional methods (Fourier transformation and fitting procedures) this method does not demand any model assumption and any special procedure of deconvolution of $L_{1}-, L_{2}-, L_{3}-$ contributions and has a high resolution in real space $r$. Obtained parameters - interatomic distance, coordination number for the first and the second coordination shells agree well with the expected crystallographic values.

\section{References}

1. Ankudinov, A., Ravel, B., Rehr, J. J. and Conradson, S., Phys. Rev. B58, 7565 (1998).

2. Tikhonov, A. N. and Arsenin, V. Ya., "Solution of Ill-Posed Problems" (John Willey and Sons, Inc., New York, 1981).

3. Babanov, Yu. A., Vasin, V. V., Ershov, N. V. and Ageev, A. L., Phys. Stat. Sol B 105, 747 (1981)

4. Babanov, Yu. A., et al., J. Non-Cryst. Sol. 79, 1 (1986).

5. Wende, H., et al., J. Appl. Phys. 83, 7028 (1998).

6. Lemke, L., et al., J. Phys. Condens. Matter 10, 1917 (1998)

7. Teo, B. K., "EXAFS: Basic Principles and Data Analysis" (Springer-Verlag, Berlin, 1986).

8. Newville, M., Livins, P., Yacoby, Y., Stern, E. A. and Rehr, J. J., Phys. Rev. B 47, 14126 (1993). 